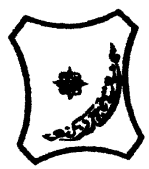

Bayero Journal of Pure and Applied Sciences, 11(1): 221 - 227

Received:

Accepted:

ISSN $2006-6996$

\title{
BAYESIAN APPROACH TO ESTIMATION OF SCALE PARAMETER OF FRECHET DISTRIBUTION
}

\author{
Hussaini G. Dikko ${ }^{1}$ and Matthew E. Isaac ${ }^{1}$ \\ ${ }^{1}$ Department of Statistics, Ahmadu Bello University, Zaria - Nigeria \\ Correspondent author: eche.isaac@yahoo.com
}

\section{ABSTRACTS}

The Frechet distribution which has a scale and shape parameters, has been found to have wide application in modelling extreme events such as radioactive emission, flood, rainfall, seismic analysis, wind speed, etc. In this research paper, the Bayesian analysis of scale parameter of Frechet distribution was considered. It is necessary to know the best combination of prior distribution and loss function for the parameter estimation. Posterior distribution was derived by uniform and Jeffrey's prior under the square error, Precautionary, Quadratic and Weighted balance loss function. Bayes estimation and their corresponding risk was obtained by the above stated priors and loss function. Monte Carlo simulations was conducted to compare the performance of the estimators. It is evident that weighted balance loss function when used with uniform prior provides the least posterior risk.

Keywords: Frechet Distribution, Non-Informative Prior, Bayesian Estimation, Loss Functions, Monte Carlo Simulations

\section{INTRODUCTION}

The Frechet distribution was named after a French mathematician Maurice Rene Frechet who developed it in the 1920 s as a maximum value distribution. The Frechet distribution is also known as extreme value distribution(EVD) Type II, it is one of the four distributions along with Gumbel distribution, Extreme value distribution and Weibull distribution classified under the general heading "extreme value distribution" and therefore used as tool for quantifying extreme events. The Frechet distribution has been shown to be useful for modeling and analyzing of several extreme events ranging from accelerated life testing to earthquakes, radioactive emissions, floods, rain fall, sea currents, horse racing, human lifespans and wind speeds; Kamran et al 2012. Harlow (2002) studied the Applications of the Frechet distribution in various fields which showed that it is an important distribution for modeling the statistical behavior of materials properties for a variety of engineering applications. Nadarajahet al (2008) discuss the sociological models based on Frechet random variables. Later, Ter (2009) study the unification of the Frechet and Weibull distribution using unifying parametrization. Also, Zaharimet al (2009) applied Frechet distribution for analyzing the wind speed data. Arora et al (2013) usedFrechet and Gumbell distribution to fit maximum annual wind speed for individual stations.

Mubarak (2011) studied the estimation of parameter of Frechet distribution under progressive type II censored data with binomial removals using maximum likelihood estimation. He also constructs confidence intervals for the parameters and percentile of the failure time distribution. Kamran et al (2012) estimated the scale parameter of Frechet distribution using Maximum Likelihood Estimation, Probability weighted moment and Bayesian estimation methods assuming known shape parameter of the Frechet distribution.

Azamet al (2014) estimated the scale parameter of Nakagami distribution under three priors; uniform, inverse exponential and levy prior and three loss functions; square error, quadratic and precautionary loss functions. Relative posterior risk and Monte Carlo simulation where used to assess the performance of the estimators. It was discovered that the precautionary loss function produces the least posterior risk when uniform prior is used while squared error loss function is best when inverse exponential prior is used.

Wajihaet al (2015) studied the shape parameter of Frechet distribution using the Gumbel Type - II and the Levy prior under four loss functions. It was observed that the Gumbel Type - II performed better than the Levy Prior as its posterior risks is smallest amongst the two assumed prior distributions. Weighted balance loss function was found to be better as its posterior risk was least among all the loss functions. So, Gumbel Type - II with weighted balance loss function provides less posterior risks as compare to other loss functions and priors.

Aliyu and Abubakar (2016) studied the estimation of shape parameter of Generalized Rayleigh distribution with assumption of non - informative prior under Squared error, Entropy and Precautionary loss functions. Comparison was also made between the performance of Maximum likelihood estimators and Bayesian estimators, and it was concluded that Bayes estimator under the entropy loss function is better than that of Squared error loss function, Precautionary loss function and that of Maximum likelihood estimation.

The cumulative distribution function (CDF) and the probability density function (PDF) for Frechet distribution are given by 


$$
F(x ; \alpha, \beta)=\exp \left[-\left(\frac{\beta}{x}\right)^{\alpha}\right],
$$

(1)

and

$$
f(x ; \alpha, \beta)=\alpha \beta^{\alpha}\left(\frac{1}{x}\right)^{\alpha+1} \exp \left[-\left(\frac{\beta}{x}\right)^{\alpha}\right], \quad x, \alpha, \beta>0
$$

where: the parameter $\alpha>0$, is the shape of the distribution and $\beta>0$ is the scale parameter.

However, in this research paper we studied the Bayes estimation of scale parameter of Frechet distribution with known shape parameter. We will use four loss function under uniform and Jeffrey prior.

\section{Loss and Risk Functions}

This section contains Bayes estimate and posterior risks of different loss functions.

In this research, we considered four loss functions. The details are given below.

\section{i. Square Error Loss Function (SELF)}

The squared error loss is defined as:

$$
L\left(\beta, \beta^{*}\right)=\left(\beta-\beta^{*}\right)^{2}
$$

where $\beta^{*}$ ( $\beta_{\text {SELF }}$ ) is the estimator of the parameter $\beta$ under SELF.

The Bayes estimator is given as

$$
\beta_{S E L F}=E(\beta \mid x)=\int_{0}^{\infty} \beta P(\beta \mid x) d \beta
$$

(4)

.and the Bayes posterior risk is obtained as:

$$
R_{\beta_{\text {SELF }}}=E\left(\beta^{2}\right)-[E(\beta)]^{2}=E\left(\beta^{2} \mid x\right)-[E(\beta \mid x)]^{2}
$$

(5)

\section{ii. Precautionary Loss Function (PLF)}

Norstrom (1996) introduced an asymmetric precautionary loss function (PLF) which is defined as:

$$
L\left(\beta^{*}, \beta\right)=\frac{\left(\beta^{*}-\beta\right)^{2}}{\beta^{*}}
$$

where $\beta^{*}\left(\beta_{P L F}\right)$ is the estimator of the parameter $\beta$ under PLF and the Bayes estimator is given as

$$
\beta_{P L F}=\left[E\left(\beta^{2} \mid x\right)\right]^{1 / 2}=\sqrt{\int_{0}^{\infty} \beta^{2} P(\beta \mid x) d \beta}
$$

(7)

The Bayes posterior risk is obtained as:

$$
R_{\beta_{P L F}}=2\left[\sqrt{E\left(\beta^{2}\right)}-E(\beta)\right]=2\left[\beta_{P L F}-E(\beta \mid x)\right]
$$

(8)

\section{iii. Quadratic Loss Function (QLF)}

The quadratic loss function can be defined as

$$
L\left(\beta, \beta^{*}\right)=\left(\frac{\beta-\beta^{*}}{\beta}\right)^{2}
$$

(9)

where $\beta^{*}\left(\beta_{Q L F}\right)$ is the estimator of the parameter $\beta$ under QLF The Bayes estimator is given as: 
$\beta_{Q L F}=\frac{E\left(\beta^{-1} \mid x\right)}{E\left(\beta^{-2} \mid x\right)}=\frac{\int_{0}^{\infty} \beta^{-1} P(\beta \mid x) d \beta}{\int_{0}^{\infty} \beta^{-2} P(\beta \mid x) d \beta}$

and

The Bayes posterior risk is gotten as:

$$
R_{\beta_{Q L F}}=1-\frac{\left[E\left(\beta^{-1} \mid x\right)\right]^{2}}{E\left(\beta^{-2} \mid x\right)}
$$

(11)

iv. Weighted Balance Loss Function (WBLF)

The weighted balance loss function can be defined as

$$
L\left(\beta, \beta^{*}\right)=\left(\frac{\beta-\beta^{*}}{\beta^{*}}\right)^{2}
$$

(12)

where: $\beta^{*}\left(\beta_{W B L F}\right)$ is the estimator of the parameter $\beta$ under WBLF

The Bayes estimator is given as:

$$
\beta_{\text {WBLF }}=\frac{E\left(\beta^{2} \mid x\right)}{E(\beta \mid x)}=\frac{\int_{0}^{\infty} \beta^{2} P(\beta \mid x) d \beta}{\int_{0}^{\infty} \beta P(\beta \mid x) d \beta}
$$

and

The Bayes posterior risk is gotten as:

$$
R_{\beta_{W B L F}}=1-\frac{[E(\beta \mid x)]^{2}}{E\left(\beta^{2} \mid x\right)}
$$

\section{Posterior Distribution}

To obtain the posterior distribution $p(\beta \mid x)$, we apply Bayes' Theorem

$$
p(\beta \mid x)=\frac{p(\beta) L(x \mid \beta)}{\int_{-\infty}^{\infty} p(\beta) L(x \mid \beta) d \beta}
$$

where $p(\beta)$ is the Prior distribution and $L(x \mid \beta)$ is the likelihood function.

\section{Likelihood Function}

Let $x_{1}, x_{2} \mathrm{~K}, x_{n}$ be a random sample of size $n$, drawn from the Frechet distribution. The Likelihood function is given as

$$
\begin{array}{r}
L(x \mid \beta)=\alpha^{n} \beta^{n \alpha} \sum_{i=1}^{n}\left(\frac{1}{x_{i}}\right)^{\alpha+1} e^{-\sum_{i=1}^{n}\left(\frac{\beta}{x_{i}}\right)^{\alpha}} \\
L(x \mid \beta) \propto \beta^{n \alpha} e^{-\sum_{i=1}^{n}\left(\frac{\beta}{x_{i}}\right)^{\alpha}}
\end{array}
$$

\section{Posterior Distribution using Uniform prior}

The Uniform Prior relating to the scale parameter is defined as:

$$
p(\beta) \propto 1 \quad 0<\beta<\infty
$$

The posterior distribution for scale parameter of Frechet distribution under uniform prior is: 


$$
p(\beta \mid x)=\frac{\beta^{n \alpha} e^{-\sum_{i=1}^{n}\left(\frac{\beta}{x_{i}}\right)^{\alpha}}}{\int_{0}^{\infty} \beta^{n \alpha} e^{-\sum_{i=1}^{n}\left(\frac{\beta}{x_{i}}\right)^{\alpha} d \beta}}
$$

which is solved by numerical integration technique.

\section{Posterior Distribution using Jefferey's Prior}

The Jeffrey's prior as non-informative prior with parameter $\beta$ is gives as

$$
\rho(\beta) \propto \frac{1}{\beta}
$$

(19)

The posterior distribution of scale parameter of Frechet distribution using Jefferey's prior is

$$
p(\beta \mid x)=\frac{\beta^{n \alpha-1} e^{-\sum_{i=1}^{n}\left(\frac{\beta}{x_{i}}\right)^{\alpha}}}{\int_{0}^{\infty} \beta^{n \alpha-1} e^{-\sum_{i=1}^{n}\left(\frac{\beta}{x_{i}}\right)^{\alpha} d \beta}}
$$

(20)

which is solved by numerical integration technique.

\section{Bayesian estimation under the assumption of different priors and loss functions}

The scale parameter of Frechet distribution has been estimated under the assumption of two non - informative priors and various loss functions including squared error loss function (SELF), quadratic loss function (QLF). Precautionary loss function(PLF) and weighted balance loss function (WBLF). The results are given in the table below.

Table 1: Bayesian estimation under the uniform and Jeffrey's priors for different loss functions

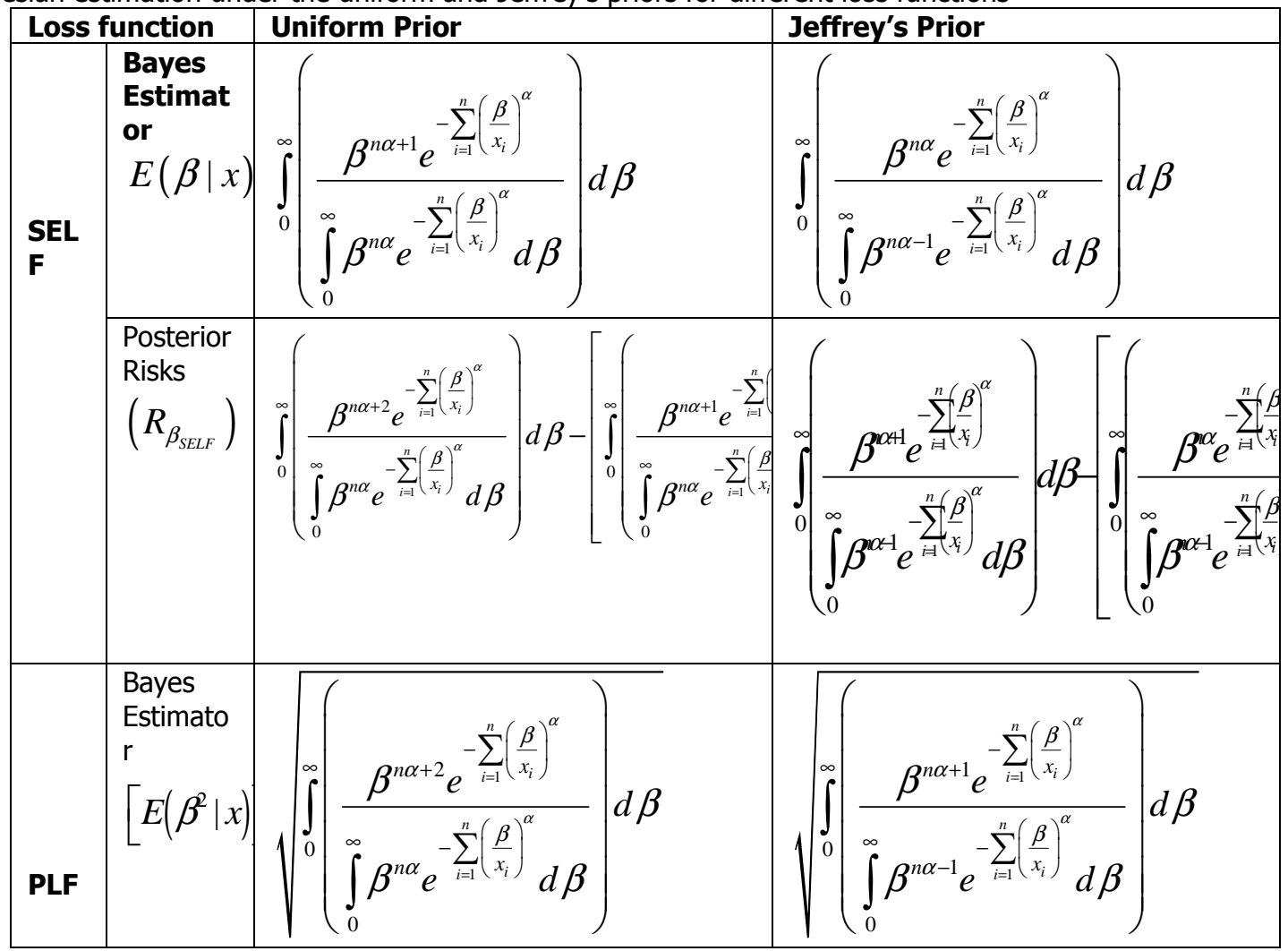




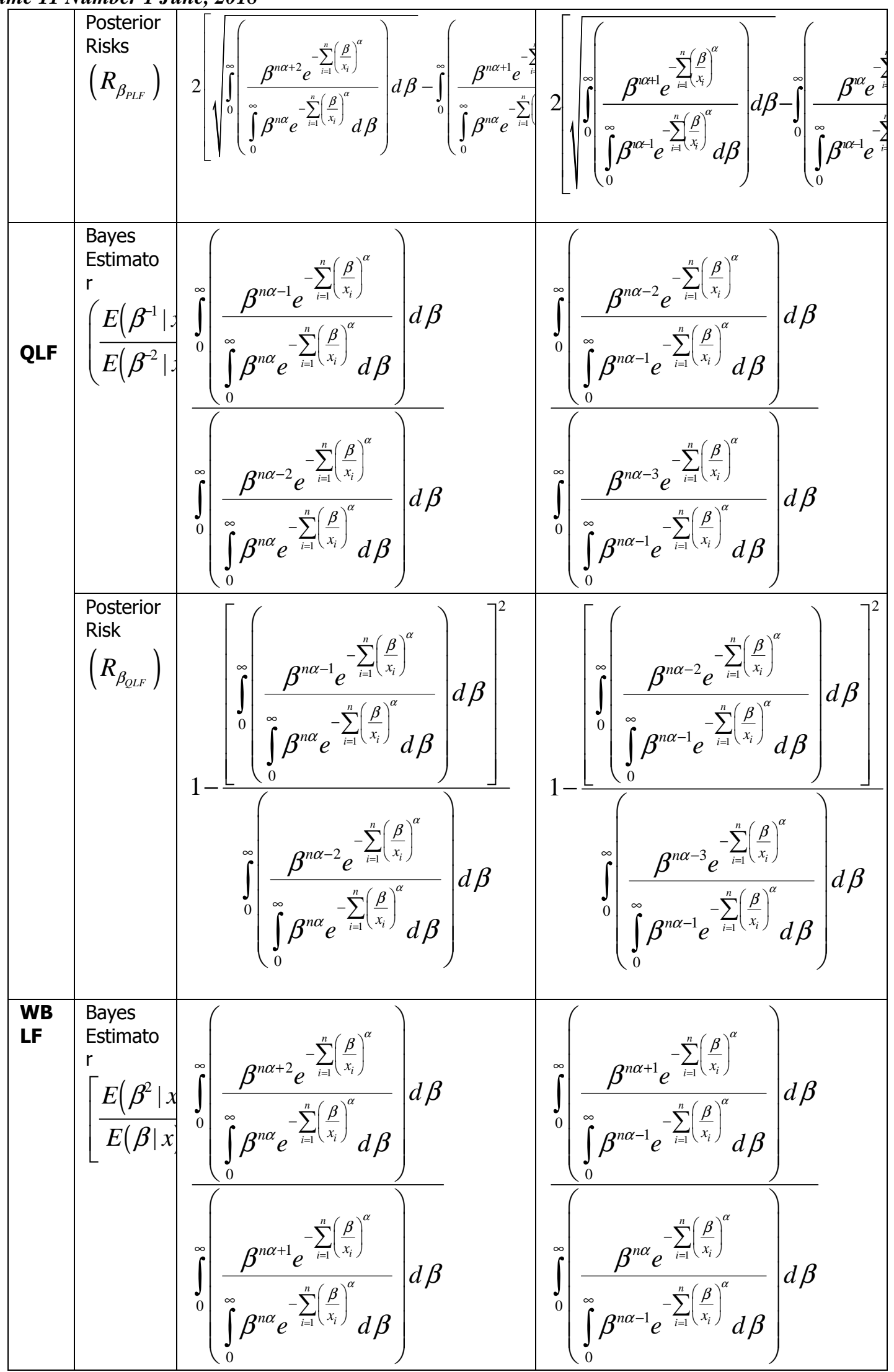




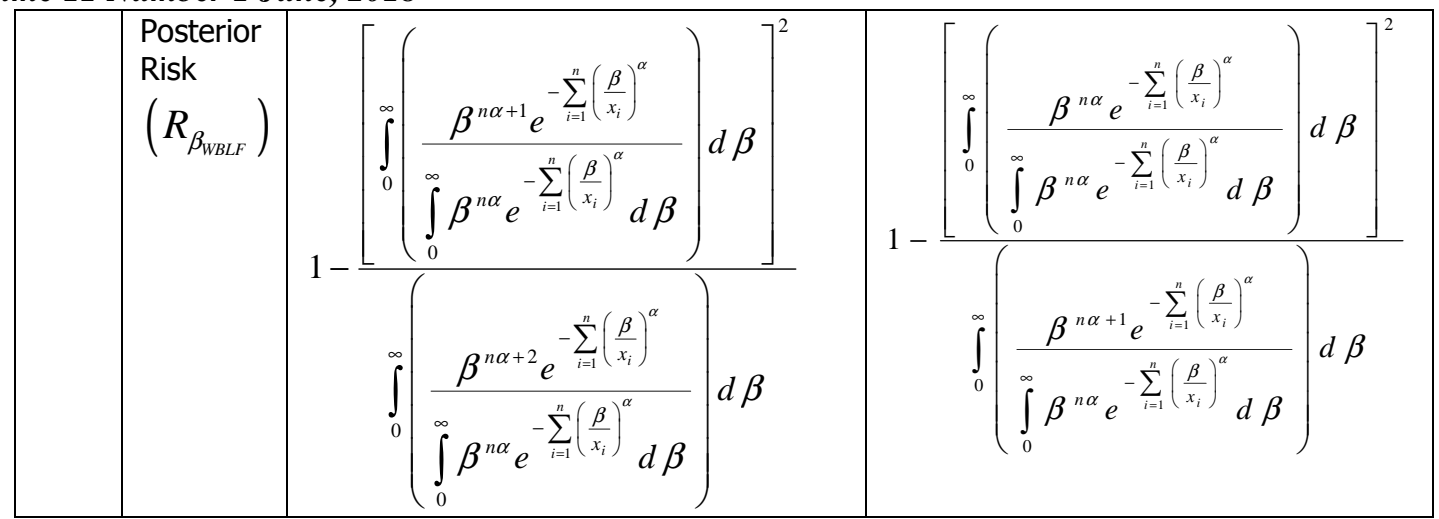

\section{Simulation Study}

We use simulation study to check the behavior and performance of prior distributions and loss functions. A comparison in terms of magnitude of posterior risks is needed to check whether an estimator is admissible under some loss function or prior distribution. We use Mathematica 11 package to generate $\mathrm{N}=1000$ random samples of size $\mathrm{n}=5,10,20,30,50,70$ and 100 from Frechet distribution using $\alpha \in(2,3)$ and $\beta \in(1.5,2)$. The Bayes posterior risks are in Parentheses. The simulation results are summarized in the following tables.

Table 2: Bayes estimates and corresponding posterior risks (within parenthesis) under different loss functions and Priors for $\alpha=2$ and $\beta=1.5$

\begin{tabular}{|c|c|c|c|c|c|c|c|c|}
\hline \multirow[t]{2}{*}{$\mathrm{N}$} & \multicolumn{4}{|c|}{ Uniform prior } & \multicolumn{4}{|c|}{ Jeffrey's prior } \\
\hline & SELF & $\begin{array}{l}\alpha=2, \\
\text { PLF }\end{array}$ & $\begin{array}{l}\beta=1.5 \\
Q L F\end{array}$ & WBLF & SELF & $\begin{array}{c}\alpha=2, \\
\text { PLF }\end{array}$ & $\begin{array}{c}\beta=1.5 \\
\mathrm{QLF}\end{array}$ & WBLF \\
\hline \multirow[t]{2}{*}{5} & 1.6556 & 1.6886 & 1.50135 & 1.7531 & 1.5795 & 1.6052 & 1.4157 & 1.6432 \\
\hline & $(0.1348)$ & $(0.0757)$ & $(0.0533)$ & $(0.0443)$ & $(0.1360)$ & $(0.0791)$ & $(0.0604)$ & $(0.0486)$ \\
\hline \multirow[t]{2}{*}{10} & 1.5994 & 1.5820 & 1.4975 & 1.6127 & 1.5475 & 1.5674 & 1.4740 & 1.5915 \\
\hline & $(0.0633)$ & $(0.0374)$ & $(0.0259)$ & $(0.0235)$ & $(0.0621)$ & $(0.0389)$ & $(0.0273)$ & $(0.0246)$ \\
\hline \multirow[t]{2}{*}{20} & 1.5324 & 1.5534 & 1.5055 & 1.5551 & 1.5109 & 1.5262 & 1.4778 & 1.5276 \\
\hline & $(0.0292)$ & $(0.0188)$ & $(0.0127)$ & $(0.0121)$ & $(0.0290)$ & $(0.0190)$ & $(0.0130)$ & $(0.0124)$ \\
\hline \multirow[t]{2}{*}{30} & 1.5252 & 1.5256 & 1.4948 & 1.5397 & 1.5176 & 1.5241 & 1.4930 & 1.5243 \\
\hline & $(0.0193)$ & $(0.0124)$ & $(0.0084)$ & $(0.0081)$ & $(0.0194)$ & $(0.0126)$ & $(0.0085)$ & $(0.0082)$ \\
\hline \multirow[t]{2}{*}{50} & 1.5234 & 1.5215 & 1.4992 & 1.5218 & 1.5102 & 1.5111 & 1.4945 & 1.5127 \\
\hline & $(0.0115)$ & $(0.0075)$ & $(0.0050)$ & $(0.0049)$ & $(0.0114)$ & $(0.0075)$ & $(0.0050)$ & $(0.0049)$ \\
\hline \multirow[t]{2}{*}{70} & 1.5130 & 1.5173 & 1.5009 & 1.5201 & 1.5071 & 1.5081 & 1.4923 & 1.5103 \\
\hline & $(0.0081)$ & $(0.0053)$ & $(0.0035)$ & $(0.0035)$ & $(0.0081)$ & $(0.0053)$ & $(0.0036)$ & $(0.0035)$ \\
\hline \multirow[t]{2}{*}{100} & 1.5083 & 1.5086 & 1.5013 & 1.5122 & 1.5034 & 1.5028 & 1.4970 & 1.5065 \\
\hline & $(0.0056)$ & $(0.0037)$ & $(0.0025)$ & $(0.0024)$ & $(0.0056)$ & $(0.0037)$ & $(0.0025)$ & $(0.0024)$ \\
\hline
\end{tabular}

Table 3: Bayes estimates and corresponding posterior risks (within parenthesis) under different loss functions and Priors for $\alpha=2$ and $\beta=2$

\begin{tabular}{|c|c|c|c|c|c|c|c|c|}
\hline \multirow[t]{3}{*}{$\mathrm{N}$} & \multirow{2}{*}{\multicolumn{4}{|c|}{$\begin{array}{l}\text { Uniform prior } \\
\alpha=2, \quad \beta=\end{array}$}} & \multirow{2}{*}{\multicolumn{4}{|c|}{ Jeffrey's prior }} \\
\hline & & & & & & & & \\
\hline & SELF & PLF & QLF & WBLF & SELF & PLF & QLF & WBLF \\
\hline 5 & $\begin{array}{c}2.2209 \\
(0.2437)\end{array}$ & $\begin{array}{c}2.2738 \\
(0.1020)\end{array}$ & $\begin{array}{c}1.9883 \\
(0.0539)\end{array}$ & $\begin{array}{c}2.3054 \\
(0.0443)\end{array}$ & $\begin{array}{c}2.0986 \\
(0.2393)\end{array}$ & $\begin{array}{c}2.1653 \\
(0.1067)\end{array}$ & $\begin{array}{c}1.8880 \\
(0.0060)\end{array}$ & $\begin{array}{c}2.2257 \\
(0.0486)\end{array}$ \\
\hline 10 & $\begin{array}{c}2.0910 \\
(0.1083)\end{array}$ & $\begin{array}{c}2.1410 \\
(0.0506)\end{array}$ & $\begin{array}{c}2.0101 \\
(0.0259)\end{array}$ & $\begin{array}{c}2.1527 \\
(0.0235)\end{array}$ & $\begin{array}{c}2.0432 \\
(0.1087)\end{array}$ & $\begin{array}{c}2.0942 \\
(0.0520)\end{array}$ & $\begin{array}{c}1.9402 \\
(0.0273)\end{array}$ & $\begin{array}{c}2.1008 \\
(0.0246)\end{array}$ \\
\hline 20 & $\begin{array}{c}2.0604 \\
(0.0528)\end{array}$ & $\begin{array}{c}2.0733 \\
(0.0252)\end{array}$ & $\begin{array}{c}2.0085 \\
(0.1273)\end{array}$ & $\begin{array}{c}2.0796 \\
(0.0121)\end{array}$ & $\begin{array}{c}2.0348 \\
(0.0528)\end{array}$ & $\begin{array}{c}2.0299 \\
(0.0252)\end{array}$ & $\begin{array}{c}1.9753 \\
(0.0130)\end{array}$ & $\begin{array}{c}2.0493 \\
(0.0124)\end{array}$ \\
\hline 30 & $\begin{array}{c}2.0389 \\
(0.0344)\end{array}$ & $\begin{array}{c}2.0426 \\
(0.0167)\end{array}$ & $\begin{array}{c}2.0010 \\
(0.0084)\end{array}$ & $\begin{array}{c}2.0535 \\
(0.0081)\end{array}$ & $\begin{array}{c}2.0194 \\
(0.0344)\end{array}$ & $\begin{array}{c}2.0256 \\
(0.0168)\end{array}$ & $\begin{array}{c}1.9753 \\
(0.0085)\end{array}$ & $\begin{array}{c}2.0366 \\
(0.0082)\end{array}$ \\
\hline 50 & $\begin{array}{c}2.0144 \\
(0.0202)\end{array}$ & $\begin{array}{c}2.0217 \\
(0.0099)\end{array}$ & $\begin{array}{c}1.9997 \\
(0.0050)\end{array}$ & $\begin{array}{c}2.0316 \\
(0.0049)\end{array}$ & $\begin{array}{c}2.0121 \\
(0.0203)\end{array}$ & $\begin{array}{c}2.0185 \\
(0.0100)\end{array}$ & $\begin{array}{c}1.9916 \\
(0.0050)\end{array}$ & $\begin{array}{c}2.0307 \\
(0.0049)\end{array}$ \\
\hline 70 & $\begin{array}{c}2.0135 \\
(0.0144)\end{array}$ & $\begin{array}{c}2.0201 \\
(0.0071)\end{array}$ & $\begin{array}{r}1.9969 \\
(0.0035)\end{array}$ & $\begin{array}{c}2.0208 \\
(0.0035)\end{array}$ & $\begin{array}{r}2.0103 \\
(0.0145)\end{array}$ & $\begin{array}{c}2.0123 \\
(0.0071)\end{array}$ & $\begin{array}{c}1.9953 \\
(0.0036)\end{array}$ & $\begin{array}{c}2.0164 \\
(0.0035)\end{array}$ \\
\hline 100 & $\begin{array}{c}2.0096 \\
(0.0100)\end{array}$ & $\begin{array}{c}2.0130 \\
(0.0052)\end{array}$ & $\begin{array}{c}2.0043 \\
(0.0025)\end{array}$ & $\begin{array}{l}2.0070 \\
(0.0024)\end{array}$ & $\begin{array}{c}2.0092 \\
(0.0101)\end{array}$ & $\begin{array}{c}2.0091 \\
(0.0050)\end{array}$ & $\begin{array}{c}1.9979 \\
(0.0025)\end{array}$ & $\begin{array}{r}2.0085 \\
(0.0024)\end{array}$ \\
\hline
\end{tabular}


Bajopas Volume 11 Number 1 June, 2018

Table 3: Bayes estimates and corresponding posterior risks (within parenthesis) under different loss functions and Priors for $=$ and $=$

\begin{tabular}{|c|c|c|c|c|c|c|c|c|}
\hline \multirow[t]{3}{*}{$\mathrm{N}$} & \multicolumn{4}{|c|}{ Uniform prior } & \multicolumn{4}{|c|}{ Jeffrey's prior } \\
\hline & & $=3$, & $=1.5$ & & & $=3$ & $=1.5$ & \\
\hline & SELF & PLF & QLF & WBLF & SELF & PLF & QLF & WBLF \\
\hline 5 & $\begin{array}{c}1.5756 \\
(0.0553)\end{array}$ & $\begin{array}{r}1.5929 \\
(0.0339)\end{array}$ & $\begin{array}{c}1.4971 \\
(0.0243)\end{array}$ & $\begin{array}{c}1.6141 \\
(0.0212)\end{array}$ & $\begin{array}{c}1.5229 \\
(0.0551)\end{array}$ & $\begin{array}{c}1.5638 \\
(0.0356)\end{array}$ & $\begin{array}{c}1.4695 \\
(0.0262)\end{array}$ & $\begin{array}{c}1.5638 \\
(0.0226)\end{array}$ \\
\hline 10 & $\begin{array}{c}1.5298 \\
(0.0260)\end{array}$ & $\begin{array}{c}1.5432 \\
(0.0168)\end{array}$ & $\begin{array}{c}1.4957 \\
(0.0116)\end{array}$ & $\begin{array}{c}1.5586 \\
(0.0108)\end{array}$ & $\begin{array}{r}1.5129 \\
(0.0263)\end{array}$ & $\begin{array}{c}1.5234 \\
(0.0171)\end{array}$ & $\begin{array}{c}1.4803 \\
(0.0120)\end{array}$ & $\begin{array}{c}1.5443 \\
(0.0112)\end{array}$ \\
\hline 20 & $\begin{array}{c}1.5154 \\
(0.0127)\end{array}$ & $\begin{array}{c}1.5231 \\
(0.0083)\end{array}$ & $\begin{array}{c}1.5410 \\
(0.0056)\end{array}$ & $\begin{array}{c}1.5304 \\
(0.0054)\end{array}$ & $\begin{array}{c}1.5081 \\
(0.0128)\end{array}$ & $\begin{array}{c}1.5117 \\
(0.0084)\end{array}$ & $\begin{array}{c}1.4923 \\
(0.0057)\end{array}$ & $\begin{array}{c}1.5193 \\
(0.0055)\end{array}$ \\
\hline 30 & $\begin{array}{c}1.5115 \\
(0.0084)\end{array}$ & $\begin{array}{c}1.5109 \\
(0.0055)\end{array}$ & $\begin{array}{c}1.4992 \\
(0.0037)\end{array}$ & $\begin{array}{c}1.5157 \\
(0.0036)\end{array}$ & $\begin{array}{c}1.5094 \\
(0.0085)\end{array}$ & $\begin{array}{c}1.5108 \\
(0.0056)\end{array}$ & $\begin{array}{c}1.4932 \\
(0.0038)\end{array}$ & $\begin{array}{c}1.5081 \\
(0.0037)\end{array}$ \\
\hline 50 & $\begin{array}{c}1.5087 \\
(0.0050)\end{array}$ & $\begin{array}{l}1.5048 \\
0.0033\end{array}$ & $\begin{array}{c}1.5001 \\
(0.0024)\end{array}$ & $\begin{array}{c}1.5099 \\
(0.0022)\end{array}$ & $\begin{array}{c}1.5051 \\
(0.0050)\end{array}$ & $\begin{array}{c}1.5033 \\
(0.0033)\end{array}$ & $\begin{array}{r}1.4949 \\
(0.0022)\end{array}$ & $\begin{array}{c}1.5081 \\
(0.0022)\end{array}$ \\
\hline 70 & $\begin{array}{c}1.5030 \\
(0.0035)\end{array}$ & $\begin{array}{c}1.5048 \\
(0.0023)\end{array}$ & $\begin{array}{c}1.4974 \\
(0.0015)\end{array}$ & $\begin{array}{c}1.5084 \\
(0.0015)\end{array}$ & $\begin{array}{c}1.5033 \\
(0.0036)\end{array}$ & $\begin{array}{c}1.5011 \\
(0.0023)\end{array}$ & $\begin{array}{c}1.4975 \\
(0.0016)\end{array}$ & $\begin{array}{c}1.5039 \\
(0.0015)\end{array}$ \\
\hline 100 & $\begin{array}{c}1.5039 \\
(0.0025)\end{array}$ & $\begin{array}{c}1.5046 \\
(0.0016)\end{array}$ & $\begin{array}{c}1.4969 \\
(0.0011)\end{array}$ & $\begin{array}{c}1.5059 \\
(0.0011)\end{array}$ & $\begin{array}{c}1.5017 \\
(0.0025)\end{array}$ & $\begin{array}{c}1.5040 \\
(0.0016)\end{array}$ & $\begin{array}{c}1.4978 \\
(0.0011)\end{array}$ & $\begin{array}{c}1.5077 \\
(0.0011)\end{array}$ \\
\hline
\end{tabular}

Table 4: Bayes estimates and corresponding posterior risks (within parenthesis) under different loss functions and Priors for $=$ and $=$

\begin{tabular}{|c|c|c|c|c|c|c|c|c|}
\hline \multirow[t]{2}{*}{$\mathrm{N}$} & \multicolumn{4}{|c|}{ Uniform prior } & \multicolumn{4}{|c|}{ Jeffrey's prior } \\
\hline & SELF & 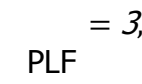 & $\begin{array}{l}=2 \\
\mathrm{QLF}\end{array}$ & WBLF & SELF & $\begin{array}{r}=3 \\
\text { PLF }\end{array}$ & $\begin{array}{l}=2 \\
\text { QLF }\end{array}$ & WBLF \\
\hline 5 & $\begin{array}{c}2.1079 \\
(0.0990)\end{array}$ & $\begin{array}{c}2.1250 \\
(0.0453)\end{array}$ & $\begin{array}{c}2.0135 \\
(0.0243)\end{array}$ & $\begin{array}{c}2.1368 \\
(0.0212)\end{array}$ & $\begin{array}{c}2.0500 \\
(0.1000)\end{array}$ & $\begin{array}{c}2.0886 \\
(0.0476)\end{array}$ & $\begin{array}{c}1.9522 \\
(0.0262)\end{array}$ & $\begin{array}{r}2.1022 \\
(0.0226)\end{array}$ \\
\hline 10 & $\begin{array}{r}2.0309 \\
(0.0460)\end{array}$ & $\begin{array}{c}2.0599 \\
(0.0224)\end{array}$ & $\begin{array}{c}2.0104 \\
(0.0116)\end{array}$ & $\begin{array}{c}2.0656 \\
(0.0108)\end{array}$ & $\begin{array}{c}2.0247 \\
(0.0471)\end{array}$ & $\begin{array}{c}2.0204 \\
(0.0227)\end{array}$ & $\begin{array}{c}1.9692 \\
(0.0120)\end{array}$ & $\begin{array}{c}3.5518 \\
(0.0501)\end{array}$ \\
\hline 20 & $\begin{array}{c}2.0164 \\
(0.0225)\end{array}$ & $\begin{array}{c}2.0207 \\
(0.0111)\end{array}$ & $\begin{array}{c}1.9920 \\
(0.0056)\end{array}$ & $\begin{array}{c}2.0342 \\
(0.0054)\end{array}$ & $\begin{array}{c}2.0167 \\
(0.0229)\end{array}$ & $\begin{array}{c}2.0067 \\
(0.0112)\end{array}$ & $\begin{array}{c}1.9832 \\
(0.0057)\end{array}$ & $\begin{array}{c}2.0295 \\
(0.0054)\end{array}$ \\
\hline 30 & $\begin{array}{c}2.0108 \\
(0.0149)\end{array}$ & $\begin{array}{c}2.0123 \\
(0.0074)\end{array}$ & $\begin{array}{c}2.0016 \\
(0.0037)\end{array}$ & $\begin{array}{c}2.0198 \\
(0.0036)\end{array}$ & $\begin{array}{c}2.0045 \\
(0.0150)\end{array}$ & $\begin{array}{c}2.0171 \\
(0.0075)\end{array}$ & $\begin{array}{c}1.9903 \\
(0.0038)\end{array}$ & $\begin{array}{r}2.0239 \\
(0.0036)\end{array}$ \\
\hline 50 & $\begin{array}{c}2.0078 \\
(0.0089)\end{array}$ & $\begin{array}{c}2.0115 \\
(0.0044)\end{array}$ & $\begin{array}{c}2.0001 \\
(0.0022)\end{array}$ & $\begin{array}{c}2.0103 \\
(0.0022)\end{array}$ & $\begin{array}{c}2.0075 \\
(0.0090)\end{array}$ & $\begin{array}{r}2.0019 \\
(0.0044)\end{array}$ & $\begin{array}{c}1.9927 \\
(0.0022)\end{array}$ & $\begin{array}{r}2.0109 \\
(0.0022)\end{array}$ \\
\hline 70 & $\begin{array}{c}2.0044 \\
(0.0063)\end{array}$ & $\begin{array}{c}2.0085 \\
(0.0031)\end{array}$ & $\begin{array}{c}2.0011 \\
(0.0015)\end{array}$ & $\begin{array}{c}2.0066 \\
(0.0015)\end{array}$ & $\begin{array}{c}2.0035 \\
(0.0064)\end{array}$ & $\begin{array}{c}2.0061 \\
(0.0031)\end{array}$ & $\begin{array}{r}1.9930 \\
(0.0016)\end{array}$ & $\begin{array}{r}2.0133 \\
(0.0015)\end{array}$ \\
\hline 100 & $\begin{array}{c}2.0026 \\
(0.0044)\end{array}$ & $\begin{array}{c}2.0071 \\
(0.0022)\end{array}$ & $\begin{array}{c}2.0019 \\
(0.0011)\end{array}$ & $\begin{array}{c}2.0078 \\
(0.0011)\end{array}$ & $\begin{array}{c}2.0047 \\
(0.0044)\end{array}$ & $\begin{array}{c}1.9998 \\
(0.0022)\end{array}$ & $\begin{array}{c}1.9968 \\
(0.0011)\end{array}$ & $\begin{array}{c}2.0061 \\
(0.0011)\end{array}$ \\
\hline
\end{tabular}

From the above tables 5.1 down to 5.4 , it is observed that the estimated value of the scale parameter approaches the true value and corresponding posterior risks associated with each estimate decreases with increase in sample size. The pattern of risks is almost similar for each prior distribution and under every loss function. It should also be noted that the magnitude of risk under the four (4) loss function are not muchly affected by the choice of prior from a sample size greater than 20 and in terms of magnitude of the parameter, it is observed that as the value of $\alpha$ increases, the magnitude of the risks decreases. Furthermore, the performance of both the QLF and WBLF are stable at various sample sizes as their posterior risks are minimal but that of WBLF is more stable. In terms of prior distributions used, the Uniform prior performs better than the Jefferey's prior22 because it estimates are associated with minimum risk when compared to the Jefferey's prior.

\section{CONCLUSION}

In this paper, Bayesian approach to estimation of scale parameter of Frechet distribution is considered. Bayes estimates and posterior risk was determined using two non - informative prior under the assumption of squared error, precautionary, quadratic and weighted balance loss function. After After conducting simulation study, comparison was made among the various Bayes estimators in terms of the magnitude of posterior risk obtained and it was observed that the estimates under uniform prior performed better than that of Jeffrey's prior as it posterior risk was smallest amongst the two-assumed prior distribution. On the other hand, weighted 6 balance lose function performs better than the other 
Bajopas Volume 11 Number 1 June, 2018

loss function as its posterior risk is least so, weight balance loss function (WBLF) under uniform prior provides less posterior risk as compared to the combination of other loss function and prior and will

\section{REFERENCES}

Aliyu, Y. and Abubakar, Y. (2016). Bayesian estimation of the shape parameter of generalized Rayleigh distribution under noninformative prior. International Journal of Advanced Statistics and Probability, 4 (1), $1-10$

Arora, D. K., Maurya, E. A. K., and Gautam, R. A. (2013). Exact modelling and analytical study of annual maximum rainfall with Gumbel and Frechet distributions using parameter estimation techniques. World of Sciences Journal, 2, 11-26.

Azam, Z., and Ahmad, S. A. (2014). Bayesian Approach in Estimation of Scale Parameter of Nakagami Distribution. Pakistan Journal of Statistics and Operations Research, $10(2), 217-228$

Harlow, D.G. (2002). Applications of the Frechet distribution function. International Journal of Material and Product Technology. 5(17): 482- 495.

Kanran, A. and Yincai, T. (2012). Comparison of Estimation method for Frechet distribution in turn provide the best efficient estimate of the scale parameter of Frechet distribution.

The study can be further extended by using informative prior distributions and some other loss functions.

with known shape. Caspian journal of Applied Science Research, 1(10); 58-64

Mubarak, M. (2011). Parameter estimation based on the Frechet progressive type ii censored data with binomial removals. International Journal of Quality, Statistics, and Reliability, 2012.

Nadarajah S, and Kotz, S. (2008). Sociological models based on Frechet random variables. Quality and Quantity, 42; 89-95.

Ter Berg, P. (2009). Unification of the Fréchet and Weibull Distribution. De Nederlandsche Bank.

Wajiha, N., and Muhammed, A. (2015). Bayes approach to study shape parameter of Frechet distribution. International Journal of Basic and Applied Sciences, 4 (3), 246 254

Zaharim A, Najidi S.K, Razali A.M. and Sopian, K. (2009), February, 24-26). Analyzing Malaysian wind speed data using statistical distribution. 